\title{
Reduction of the Self-dual Yang-Mills Equations to Sinh-Poisson Equation and Exact Solutions
}

\author{
GHARIB .M.GHARIB, RANIA SAADEH \\ Mathematics Department, College of Science \\ Zarqa University, \\ JORDAN
}

\begin{abstract}
The geometric properties of differential systems are used to demonstrate how the sinh-poisson equation describes a surface with a constant negative curvature in this paper. The canonical reduction of 4dimensional self dual Yang Mills theorem is the sinh-poisson equation, which explains pseudo spherical surfaces. We derive the Bäcklund transformations and the travelling wave solution for the sinh-poisson equation in specific. As a result, we discover exact solutions to the self-dual Yang-Mills equations.
\end{abstract}

KeyWords :- Bäcklund transformations; Nonlinear evolution equations; Yang-Mills theory; Pseudospherical surfaces.

Received: May 13, 2021. Revised: September 24, 2021. Accepted: October 6, 2021. Published: October 26, 2021.

\section{Introduction}

The concept of integrable structures has been a contentious issue in mathematics for the last three decades. The focus includes but not limited into mechanics, dynamics, mathematics, algebra, physics, evaluation, and geometry.

Many partial differential equations, which are still studied due to their significance in physics and mathematics, have relations with the geometry of surfaces embedded in 3D space [1]. This has long been known that there is a relationship between surfaces in Euclidean three-space with a constant negative Gaussian curvature, the SineGordon Formula, and Bäcklund transformations that are applicable to the equation given [2]. Furthermore, including pseudo spherical surfaces, the main Bäcklund transformation for something like the SineGordon Formula appears to have been a normal linear structure (pss) [3-5].

The Yang-Mills (YM) behavior is one of the key components of the standard model, which has been phenomenological very popular up to this point. A Yang-Mills field adds to the curvature of space-time in the same way as every other field does, according to General Relativity. There are some physically important circumstances in which gravitational fields are exceptionally strong, and the impact of curvature on the propagation of matter fields, as well as the Yang-Mills fields' back-reaction, cannot be overlooked [6].

Once Yang-Mills concept was proposed in the midtwentieth century, it was understood that the quantum model of the Maxwell system, known as
Quantum Electrodynamics (QED), provided a detailed explanation of the quantum structure of electro_magnetic fields and forces. The issue of whether or not the nonabelian analog was needed to represent other natural forces, especially the weak force, which is concerned with certain types of radioactivity, and the powerful or nuclear force, which is concerned with the mixing of protons and neutrons around nuclei, among other things, arose. Yang-Mills theories, which describe fundamental laws of interactions, are central to primary particle physics.Topological solitons such as instantons, monopoles, vortices, calorons, and merons played important roles in the study of non-perturbative properties, duality structures, and quark con nements, among other aspects, in these theories.

Since the weak and nuclear forces are unrelated to long-range fields or particles of low mass [7-9]. The masslessness of classical Yang-Mills waves was a major impediment to applying Yang-Mills theory to them. In the 1960s and 1970s, these obstacles to physical applications of non-abelian gauge theory were overcome.

The self-dual Yang-Mills equations (SDYM) are well-known for having a large number of integrable systems. The Painleve equations and classical soliton equations in $1+1$ dimensions are finitedimensional Lie algebra reductions of the SDYM equations (LG). It is important to use infinite dimensional algebras to minimize the SDYM equations [10-12].

Anti-self-dual Yang-Mills(ASDYM) equation, on the other side, has a close relationship with lower- 
dimensional integrable equations such as $\mathrm{KdV}$,Toda, and Painlev equations, and so on [18, 21]. Any soliton solution towards these equations has energy densities that are local on hyper - plane over all three dimensions, allowing them to be viewed as space-time domain walls. Even so, the integrability of those kind of solitons systems is dependent on the presence of infinite quantities and undefined infinite transformations.

The objective of this research is to propose and exact solutions to the canonical reduction of 4D SDYM theory to 2D sinh-poisson (SP) equation. T he section is structured as follows. Section 2 establishes the relationship in between SP equation and pss. Sections 3 and 4 look at the canonical reduction of 4D SDYM to the above equation. In addition, the travelling wave solution for the SP equation and the gauge potential $A \mu$ are obtained. Section 5 concludes with certain assumptions.

\section{Description of Pseudo-Spherical Surface by Sinh-Poisson Equation}

The definition of pss first appeared in geometry in the far past. That was a watershed point in the history of mathematics. A pss is the final element in the visual representation of non-Euclidean hyperbolic geometry, according to Klingenberg [13]. Pss was realized to have a close relationship with many theorems, such as theory of solitons, nets, attractors, a lot of nonlinear evolution equations (NLEEs) of mechanics, BTs, and other advanced approaches in mathematics [14-16]. For a long time, mathematical physics has studied the relationship between geometry and nonlinear partial differential equations (NLPDEs). For example, the classic Liouville equation indicates minimum surfaces in space E3, while the sine Gordon equation is identical to the geometry of pss, or surfaces with a negative Gaussian curvature $[17,18]$.

A scalar differential equation (DE) $f\left(x, t, G, G_{x}, \ldots, G_{x^{n}} t^{m}\right)=0, G_{x^{n} t^{m}}=\frac{\partial^{n+m} u}{\partial \partial t^{m}}$, (1)

Consequently, if $F\left(x, t, G, G_{x}, \ldots, G_{x^{n} t^{m}}\right)=0$ describe pss with associated one-forms $y i$ (2). A solution $G(x, t)$ of $F\left(x, t, G, G_{x}, \ldots, G_{x^{n} t^{m}}\right)=0$ is

I-generic if $(\gamma 3 \wedge \mathrm{\gamma} 2)(G(\mathrm{\gamma}, t)) \neq 0$; II-generic if $(\gamma 1 \wedge \gamma 3)(G(\gamma, t)) \neq 0$; and III-generic if $(\gamma 1 \wedge$ $\left.\gamma^{2}\right)(G(x, t)) \neq 0$.
The smooth functions fij, $1 \leq i \leq 3,1 \leq j \leq 2$, depending on $G(x, t)$ and its derivatives, one-forms [5]

$\mathrm{\gamma}_{i}=f_{i 1} d x+f_{i 2} d t, \quad 1 \leq i \leq 3$,

The constant Gaussian curvature $=-1$, that is

$d \mathrm{\gamma}_{1}=\mathrm{\gamma}_{3} \Lambda \mathrm{\gamma}_{2}, d \mathrm{\gamma}_{2}=\mathrm{\gamma}_{1} \Lambda \mathrm{\gamma}_{3}, d \mathrm{\gamma}_{3}=\mathrm{\gamma}_{1} \Lambda \mathrm{\gamma}_{2}$. (3)

It is equivalent to say that the NLEE for $G(x, t)$ is necessary and sufficient for the integrality of the linear system [18]

$\mathrm{d} \varphi=\propto \varphi, \varphi=\left(\begin{array}{l}\varphi_{1} \\ \varphi_{2}\end{array}\right), \alpha=\frac{1}{2}\left(\begin{array}{cc}\gamma_{2} & \gamma_{1}-\gamma_{3} \\ \gamma_{1}+\gamma_{3} & -\gamma_{2}\end{array}\right)(4)$

Where d denotes exterior differentiation, then

$\alpha=\left(\begin{array}{cc}\frac{f_{21}}{2} d x+\frac{f_{22}}{2} d t & \frac{\left(f_{11}-f_{31}\right)}{2} f x+\frac{\left(f_{12}-f_{32}\right)}{2} d t \\ \frac{\left(f_{11}-f_{31}\right)}{2} f x+\frac{\left(f_{12}-f_{32}\right)}{2} d t & -\frac{f_{21}}{2} d x+\frac{f_{22}}{2} d t\end{array}\right)=$ $H d x+L d t$

(5)

Eqs. (4), (5), we comes

$\varphi_{x}=H \varphi, \varphi_{t}=L \varphi$,

where $\mathrm{H}$ and $\mathrm{L}$ are two $2 \times 2$ null-trace matrices

$H=\left(\begin{array}{cc}\frac{f_{21}}{2} & \frac{f_{11}-f_{31}}{2} \\ \frac{f_{11}+f_{31}}{2} & -\frac{f_{21}}{2}\end{array}\right)$,

(7)

$L=\left(\begin{array}{cc}\frac{f_{22}}{2} & \frac{f_{12}-f_{32}}{2} \\ \frac{f_{12}+f_{32}}{2} & -\frac{f_{22}}{2}\end{array}\right)$.

(8)

Now

$d^{2} \varphi=d \alpha \varphi-\alpha \Lambda d \varphi=(d \alpha-\alpha \Lambda \alpha) \varphi$,

which

$\Theta \equiv d \alpha-\alpha \Lambda \alpha=0$,

(9)

or in component form

$f_{12, x}-f_{11, t}=f_{31} f_{22}-f_{21} f_{32}$,

(10)

$f_{22, x}-f_{21, t}=f_{11} f_{32}-f_{12} f_{31}$,

(11)

$f_{32, x}-f_{31, t}=f_{11} f_{22}-f_{21} f_{12}$,

(12) 
The SP equation describe pss as well. The equation has many physical applications $[2,4]$.

Example: Let a differentiable surface $M^{2}$,

The SP equation

$\mathrm{\gamma}_{1}=G_{x} d x$,

$\mathrm{\gamma}_{2}=\eta d x+\frac{1}{\eta} \cosh G d t$

(13)

$\mathrm{\gamma}_{3}=\frac{1}{\eta} \sinh G d t$

Hence $M^{2}$ is a pss iff $G$ satisfies the $S P$ equation $\mathrm{G}_{\mathrm{xt}}=\sinh \mathrm{G}$.

(14)

\section{Reducing "SDYM" Equations to "SP" Equation}

The SDYM equations are particularly significant in their own right, with wide range of applicable issues in science of math besides physics. SDYM equations is being used in gauge principle and classical quantum theory, and they're used to efficiently analyze four-manifolds [19, 20]. The SDYM equations are important in analyzing of integrable systems, as well as in plenty other aspects of math and physics. The master integrable scheme is another name for SDYM equations.

SDYM include a feature for developing and categorizing a large number of integrable structures, as well as a geometrical framework for analyzing them. Furthermore, an integrable equation considers comprehensive solution achieved by resolving related linear problems using the inversed scattering transformation [21-34].

The equations of SDYM are a set of 4D paired with 2nd order partial differential equations (PDEs) for Lie algebras defined gauge potential functions

$A_{\mu}{ }^{\prime} S(\mu=0,1,2,3)$, which are notoriously difficult to solve in general.

${ }^{*} F=\lambda F$,

for some constant $\lambda,{ }^{*} F$ is the dual twoform of $F$. [1-3] , the SDYM equation

$F_{01}=F_{23}, F_{02}=F_{31}, F_{03}=F_{12}$,

(16)

where $F=\frac{1}{2} F_{\mu \nu} d x^{\mu} d x^{\nu}$. Introduce the null coordinates $y, \bar{y}, z, \bar{z}$ Eq. (16)is cast into

$F_{y z}=0, F_{\overline{y z}}=0, F_{y \bar{y}}+F_{z \bar{z}}=0$,

(17) where

$y=\frac{1}{\sqrt{2}}\left(x_{1}+i x_{2}\right), \bar{y}=\frac{1}{\sqrt{2}}\left(x_{1}+i x_{2}\right)$,
$z=\frac{1}{\sqrt{2}}\left(x_{0}+i x_{3}\right), \bar{z}=\frac{1}{\sqrt{2}}\left(x_{0}+i x_{3}\right)$.

(18)

It is verified that Eq. (16) is the compatibility of the following isospectral problem:

$\left(\partial_{y}+\xi \partial_{\bar{z}}\right) v=\left(E_{y}+\xi E_{\bar{z}}\right) v,\left(\partial_{z}+\xi \partial_{\bar{y}}\right)$

$v=\left(E_{z}+\xi E_{\bar{y}}\right) v$,

(19)

where

$E=E_{\mu} d x^{\mu}=E_{y} d y+E_{\bar{y}} d \bar{y}+E_{z} d z+$

$E_{\bar{z}} d \bar{z} \xi$.

We see that

$E_{y z}-E_{z y}-\left[E_{y}, E_{z}\right]=0$,

$E_{y \bar{y}}+E_{z \bar{z}}-E_{\bar{z} z}-E_{\bar{y} y}-\left[E_{y}, E_{\bar{y}}\right]-$

$\left[E_{z}, E_{\bar{z}}\right]=0$,

(20)

$E_{\overline{y z}}-E_{\overline{z y}}-\left[E_{\bar{y}}, E_{\bar{z}}\right]=0$,

where

$E_{\mu v}=\partial_{\mu} E_{v}, F_{\mu v}=\partial_{\mu} E_{v}-\partial_{v} E_{\mu}-\left[E_{\mu}, E_{v}\right]$

and

$E_{0}=\frac{1}{\sqrt{2}}\left(E_{z}+E_{\bar{z}}\right), E_{1}=\frac{1}{\sqrt{2}}\left(E_{y}+E_{\bar{y}}\right)$,

$E_{2}=\frac{i}{\sqrt{2}}\left(E_{y}+E_{\bar{y}}\right), E_{3}=\frac{-i}{\sqrt{2}}\left(E_{z}+E_{\bar{z}}\right)$

(21)

In the end of this section, we discuss reduction of the SDYM equations to integrable PDE, many integrable equations are reductions of the SDYM ,As this application, a good NLEE could be obtained in the following example:

\subsection{The SPEquation}

Suppose that $E_{\mu}^{\prime} s$ which $E_{\bar{y}}=0$, then the equation (19) becomes

$\partial_{x} v=\left(E_{y}+\xi E_{\bar{z}}\right) v, \partial_{t} v=-\frac{1}{\xi} E_{z} v$

(22)

The $E_{\mu}^{\prime} \quad s$ are skew-Hermitian. Then the parameterization

$E_{y}=\left(\begin{array}{cc}\frac{-\mathrm{ik}}{2} & 0 \\ 0 & \frac{\mathrm{ik}}{2}\end{array}\right), \mathrm{E}_{\mathrm{z}}=\left(\begin{array}{cc}0 & \frac{-\mathrm{m}+\mathrm{m}}{2} \\ \frac{-\mathrm{m}+\mathrm{m}}{2} & 0\end{array}\right)$, 
$\mathrm{E}_{\overline{\mathrm{z}}}=\left(\begin{array}{cc}0 & \frac{\mathrm{i}}{2} \\ \frac{\mathrm{i}}{2} & 0\end{array}\right)$,

(23)

where $k, m . n$ are real functions. And SDYM equivalent to

$\frac{\partial n}{\partial x}=m k, \frac{\partial m}{\partial x}=n k, \frac{\partial k}{\partial t}=m$.

(24)

It follows from the fi two equations of (20) that $n^{2}-m^{2}$ is independent of $x$. We choose $n^{2}-m^{2}$ $=1$ and introduce the paramertization $m=\sinh$ $G, n=\cosh G$. this gives $k=\frac{\partial u}{\partial x}$ The third equation in (20) then becomes the SP equation (14).

\section{Exact Solutions of SP Equation and the SDYM Equations}

The problem [3], with Gaussian curvature $=-1$ on $M^{2}$ take

$\alpha=\left(\begin{array}{cc}\frac{\eta}{2} d x+E d t & q d x+B d t \\ r d x+C d t & -\frac{\eta}{2} d x-E d t\end{array}\right)=H d x+L d t$,

where $H$ and $L$ are two $2 \times 2$ null-trace matrices

$H=\left(\begin{array}{cc}\frac{\eta}{2} & q \\ r & -\frac{\eta}{2}\end{array}\right)$,

$$
L=\left(\begin{array}{cc}
E & B \\
C & -E
\end{array}\right) \text {, }
$$

Here $\eta$ is a parameter, independent of $x$ and $t, q$ and $r$ are functions of $x$ and $t$.

The equations (10) - (12) become,

$E_{x}=q C-r B$,

$q_{t}=-2 E q-B_{x}+\eta B=0$,

(28)

$C_{x}=r_{t}+2 E r-\eta C$,

and Tenenblat [5] obtained equations (28) directly from the structure equations (25). By suitably choosing $r, E, B$ and $C$ in (28), we shall obtain various NLEEs which $q$ must satisfy. From equations (4)-(6), we obtain the following scattering problem

$\varphi_{1 x}=\frac{\eta}{2} \varphi_{1}+q \varphi_{2}$, $\varphi_{2 x}=r \varphi_{1}+\frac{\eta}{2} \varphi_{2}$

(29)

$\varphi_{1 t}=E \varphi_{1}+B \varphi_{2}$,

$\varphi_{2 t}=C \varphi_{1}+E \varphi_{2}$

(30)

Konno and Wadati [14] introduced the function $\Gamma=\frac{\varphi_{1}}{\varphi_{2}}$,

(31)

where the function $\Gamma$ determines geodesic coordinates on the pss determined by $G_{t}=F$ $\left(G, G_{X}, \ldots, G_{x^{k}}\right)\left(G_{x^{k}}=\partial^{k} G / \partial x^{k}\right)[15]$. Then equations (29), (30) are reduced to the Riccati equations:

$\frac{\partial \Gamma}{\partial x}=\eta \Gamma-r \Gamma^{2}+q$,

(32)

$\frac{\partial \Gamma}{\partial t}=2 E \Gamma-C \Gamma^{2}+B$,

(33)

Our procedure in the following is that we construct a transformation $\Gamma^{\prime}$ satisfying the same equation as (32) with a potential $u^{\prime}$ where

$u^{\prime}=u+f(\Gamma, \eta)$,

(34)

For any solution $G(x, t)$ of the SP equation (14), the matrices $H$ and $L$ are

$H=\left(\begin{array}{cc}\frac{\eta}{2} & \frac{u_{x}}{2} \\ \frac{u_{x}}{2} & -\frac{\eta}{2}\end{array}\right)$

$L=\left(\begin{array}{ll}\frac{1}{2}\left(\frac{\cosh u}{\eta}\right) & -\frac{1}{2}\left[\frac{\sinh u}{\eta}\right] \\ \frac{1}{2}\left[\frac{\sinh u}{\eta}\right] & -\frac{1}{2}\left(\frac{\cosh u}{\eta}\right)\end{array}\right)$

the above matrices $H, L$ satisfy the equation (28). Then equation (32) becomes

$\frac{\partial \Gamma}{\partial x}=\eta \Gamma+\frac{1}{2} u_{x}\left(1-\Gamma^{2}\right)$.

(37)

If we choose $\Gamma^{\prime}$ and $u^{\prime}$ as

$\Gamma^{\prime}=\frac{1}{\Gamma}$

(38) $u^{\prime}=$

$-u+4 \tanh ^{-1} \Gamma$,

then $\Gamma^{\prime}$ and $u^{\prime}$ satisfies equation (37).

Substitute $G=G_{0}$ into the matrices $H$ and $L$ in (35) and (36), then by (6) we have 
$d \varphi=\varphi_{x} d x+\varphi_{t} d d t=H \varphi d p$

(40)

Where

$H=\left(\begin{array}{cc}\frac{\eta}{2} & 0 \\ 0 & -\frac{\eta}{2}\end{array}\right)$,

(41)

$p=x+k t, k=\frac{-\cosh u_{0}}{\eta^{2}}$,

The solution of equation (40) is

$\varphi=e^{\rho P} \varphi_{0}=\left(I+\rho P+\frac{\rho^{2} p^{2}}{2 !}+\frac{\rho^{3} p^{3}}{3 !}+\right) \varphi_{0}$,

where $\emptyset_{0}$ is a constant column vector. The solution of equation (43) is

$\varphi\left(\begin{array}{cc}\cosh \frac{\eta}{2} \rho+\sinh \frac{\eta}{2} \rho & 0 \\ 0 & \cosh \frac{\eta}{2} \rho-\sinh \frac{\eta}{2} \rho\end{array}\right)$

$\varphi_{0}$,

(44)

Now, we choose $\varphi_{0}=(1,1)^{T}$ in $(44)$, then we have

$\varphi=\left(\begin{array}{l}\frac{\eta \rho}{e^{2}} \\ \frac{\eta \rho}{e^{2}}\end{array}\right)$.

(45)

Substitute (45) into (39), then by (31), we obtain the new solutions of the SP equation (14)

$u^{\prime}=u_{0}+4 \tanh ^{-1}\left(e^{\eta \rho}\right) \rho=x-\frac{\cosh u_{0}}{\eta^{2}} t$

(46)

Consequently we obtain the gauge potential $E_{\mu}$ as follows:

$E_{\bar{y}}=0$,

$E_{y}=\left(\begin{array}{cc}\eta i \operatorname{csch}(\eta \rho) & 0 \\ 0 & -\eta i \operatorname{csch}(\eta \rho)\end{array}\right)$,

$E_{z}=\left(\begin{array}{cc}0 & \frac{a}{2} \\ \frac{a}{2} & 0\end{array}\right), \quad E_{\bar{z}}=\left(\begin{array}{cc}0 & \frac{i}{2} \\ \frac{i}{2} & 0\end{array}\right)$,

(47)

Where

$a=-\sinh \left[-u_{0}+4 \tanh ^{-1}\left(e^{\eta \rho}\right)\right]+$

$i \cosh \left[-u_{0}+4 \tanh ^{-1}\left(e^{\eta \rho}\right)\right]$

\section{Conclusions}

Since the fundamental laws of quantum mechanics, gravity, electromagnetism, and elementary particle interactions are all clarified in the manner of partial differential equations (PDEs), science has produced a great success in physics in which partial differential equations contribute significantly. In exchange, advances in physics include immense opportunities, motivation, and obstacles, all of which have fueled research in partial differential equations. We will hope to discover a relationship between the SP equation and the pss in this paper. The SDYM equations are fundamental in many fields of mathematics and physics, as well as in the field of integrable systems. The reality that indeed SDYM equations are the compatible scenario of a relevant linear problem, which accepts enormous flexibility if the involved gauge algebra(LG) is random, implies that they are a valuable source of integrable models. The SDYM equations are finite-dimensional gauge algebra reductions of the classic soliton equations in $1+1$ dimensions. Our study shows how the SDTM equations can be reduced to SP equations that explain pss and precise solutions.

\section{References:}

[1] Ablowitz,M.J.,Chakravarty ,S. and Halburd,R.,Integrable systems and reductions of the self-dual Yang-Mills equations ,J.Math.Phys.44,3147(2003)3147-3174.

[2] Abolwitz,M.J.and Clarkson,P.A., Solitons , Nonlinear Evolution Equations and Inverse Scattering (Cambridge University Press, Cambridge, 1991).

[3] Ablowitz,M.J.,Kaup,D.J.,Newell ,A.C.and Segur,H.,The inversescatter-ingtransformFourieranalysisfornonlinearproblems,Stud.A ppl.Math.53,(1974)249-256.

[4] Chadan,K.and Sabatier,P.C.,Inverse problem in quantum scattering theory (springer,NewYork,1977).

[5] Chern,S.S.and Tenenblat ,K.,Pseudo spherical surfaces and evolution equations,Stud.Appl.Math.74,(1986)1-10.

[6] Felsager,B.,Geometry , Particles and 
Fields,Berlin,NewYork:Springer-Verlag, 1998.

[7] Khater,A.H.,Callebaut,D.K., Abdalla,A.A.and Sayed,S.M.,Exact solutions for self-dual Yang-Mills Equations .Chaos Solitons\&Fractals, 10(1999)13091320.

[8] Khater,A.H.,Callebaut,D.K.,Abdalla,A.A.,S hehata,A.M.andSayed,S.M.,BäcklundTransformations and Exact solutions for self-dual SU(3)Yang-Mills Equations ,ILNuovo CimentoB114(1999)1-10.

[9] Khater,A.H.and Sayed,S.M.,Exact Solutions for Self-Dual SU(2) and SU(3)Yang-Mills Fields. International J. of Theoretical Physics,41(2002)409-419.

[10] Khater,A.H., Shehata ,A.M., Callebaut , D.K. and Sayed ,S.M .,Self-Dual Solu-tions for SU(2) and SU(3) Gauge Fields on Euclidean Space. International J. of Theoretical Physics,43(2004)151-159.

[11] Khater,A.H.,Callebaut,D.K.and Sayed ,S.M.,Conservationl aws for some nonlinear evolution equations which describe pseudospherical surfaces ,Journal of Geometry and Physics 51(2004)332-352.

[12] Khater,A.H.,Callebaut,D.K. and Sayed ,S.M.,Exact solutions for some non-linear evolution equa-tions which describe pseudospherical surfaces, Journal of Computational and Applied Mathematics,189 (2006) 387411.

[13] Klingenberg,W.,Riemannian

Geometry, Walterde

Gruyter,Berlin,NewYork,1982.

[14] Konno ,K .and Wadati,M.,Simpled erivation of Bäcklund transformation from Riccati form of inverse method ,Progr. Theoret. Phys.53,(1975)1652-1655.

[15] Sayed,S.M.,The Bäcklund Transformations ,Exact Solutions , and Conserva-tion Laws for the Compound Modifi KortewegdeVries-Sine-GordonEqua-tions which Describe Pseudo spherical Surfaces ,Journal of Applied Mathematics Volume 2013,http://dx.doi.org/10.1155/2013/613065

[16] Sayed,S.M.and Gharib ,G.M.,Canonicalr eduction of Self-DualYang-Mills equation sto Fitzhugh-Nagumo equation and exact solutions ,Chaos Solitons \& Fractals 39(2009)492-498.

[17] Sayed,S.M.,Elhamahmy,O.O. and Gharib, G.M., Travelling Wave Solutions for the KdV-Burgers-Kuramoto and Nonlinear Schrödinger Equations Which De-scribe Pseudo spherical Surfaces, Journal of Applied Mathematics Volume 2008,doi:10.1155/2008/576783.

[18] Sayed ,S.M., Elkholy ,A.M. and Gharib,G.M.,Exact solutions and conservationl aws for Ibragimov-Shabat equation which describe pseudo-spherical surface ,Computational\&Applied Mathematics ,27(2008)305-318.

[19] Tarantello ,G.,Self-dual gauge fivortices ,Progress in nonlinear difftial equations and their applications 72,Boston:Birkhäuser,2008.

[20] Yang,C.N. and Mills ,R.L. ,Conservation of isotopics in and isotopic gauge in variance ,Phys.Rev.96,(1954)191-206.

[21] Zachos,C.,Fairlie,D.and Curtrigh ,T., Quantum Mechanics in phase space,

[22] Paul, C. and Olivier, F. (2017) On the Hamiltonian Structure of Large Deviations in Stochastic Hybrid Systems. Journal of Statistical Mechanics: Theory and Experiment, 2017, 534542.

[23] Donagi, R. and Witten, E. (1996) Super symmetric Yang-Mills Theory and Integrable Systems. Nuclear Physics B , 460, 299304.https://doi.org/10.1016/05503213(95)00609-5.

[24] Reyes, E.G. (2000) Conservation Laws and Calapso-Guichard Deformations of Equations Describing Pseudo-Spherical Surfaces. Journal of Mathematical Physics, 41, 2968-2979. https://doi.org/10.1063/1.533284.

[25] Atiyah, M.F. and Ward, R.S. (1977) Instants and Algebraic Geometry. Communications in Mathematical Physics, 55, 117-129. https://doi.org/10.1007/BF01626514.

[26] Chakravarty, S. and Halburd, R. G., "First integrals of a generalized Darboux-Halphen system,'’ J. Math. Phys. 44,1751-1762 2003. 
[27] M. S. Volkov and D. V. Gal'tsov, Phys. Rept. 319, 1 (1999) [hep-th/9810070].

[28] F. Canfora, A. Giacomini and J. Oliva, Phys. Rev. D 82, $045014 \quad$ (2010) [arXiv:1004.2718[hep-th]].

[29] Zhang Yufeng. A complex higher-dimensional Lie algebra with real and imaginary structure constants as well as its decomposition. Common Theory Phys 2008;50:1021-6.

[30] A. Anabalon, F. Canfora, A. Giacomini and J. Oliva, Phys. Rev. D 83, 064023 (2011) [arXiv:1012.3209 [hep-th]].

[31] T. BERGER, T. REIS, AND S. TRENN, Observability of linear differential-algebraic systems: a survey, in Surveys in DifferentialAlgebraic Equations IV, Springer, 2017, pp. 161-219.

[32] T. BERGER AND S. TRENN, The quasiKronecker form for matrix pencils, SIAM Journal on Matrix Analysis and Applications, 33 (2012), pp. 336-368.

[33] G.M. Gharib, Conserved Quantities and Fluxes for Some Nonlinear Evolution Equations, WSEAS TRANSACTIONS on MATHEMATICS DOI: 10.37394/23206.2020.19.51.

[34] DURAND, PHILIPPE. "From QFT to 2-d Supersymetric TQT: Mirror symmetry between Math and Physic."WSEAS TRANSACTIONS on MATHEMATICS,
Contribution of Individual Authors to the Creation of a Scientific Article (Ghostwriting Policy)

All authors contribute equally in this project.

Sources of Funding for Research Presented in a Scientific Article or Scientific Article Itself

This research is funded by the Deanship of Scientific Research in Zarqa University /Jordan.

\section{Creative Commons Attribution License 4.0} (Attribution 4.0 International, CC BY 4.0) This article is published under the terms of the Creative Commons Attribution License 4.0 https://creativecommons.org/licenses/by/4.0/deed.en _US

DOI: $10.37394 / 23206.2020 .19 .51$ 